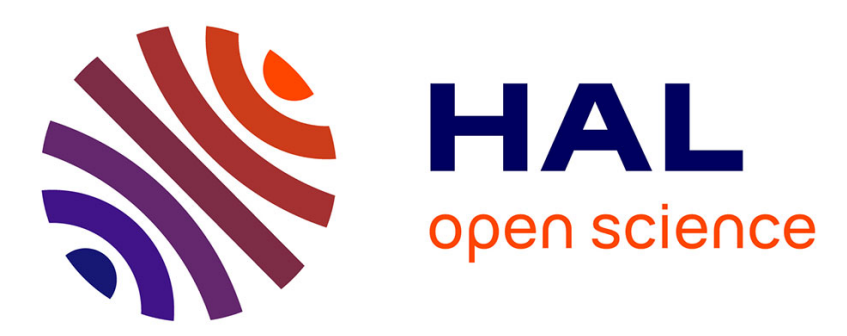

\title{
Change in the pulse component separation for PSR $\mathrm{J} 1906+0746$
}

G. Desvignes, Ismaël Cognard, M. Kramer, A. Lyne, B. Stappers, Gilles Theureau

\section{> To cite this version:}

G. Desvignes, Ismaël Cognard, M. Kramer, A. Lyne, B. Stappers, et al.. Change in the pulse component separation for PSR J1906+0746. 40 YEARS OF PULSARS: Millisecond Pulsars, Magnetars and More, Aug 2007, Montréal, Canada. pp.482, 10.1063/1.2900280 . insu-02778058

\section{HAL Id: insu-02778058 \\ https://hal-insu.archives-ouvertes.fr/insu-02778058}

Submitted on 31 Aug 2020

HAL is a multi-disciplinary open access archive for the deposit and dissemination of scientific research documents, whether they are published or not. The documents may come from teaching and research institutions in France or abroad, or from public or private research centers.
L'archive ouverte pluridisciplinaire HAL, est destinée au dépôt et à la diffusion de documents scientifiques de niveau recherche, publiés ou non, émanant des établissements d'enseignement et de recherche français ou étrangers, des laboratoires publics ou privés. 


\title{
Change in the pulse component separation for PSR J1906+0746
}

\author{
G. Desvignes*, I. Cognard*, M. Kramer ${ }^{\dagger}$, A. Lyne ${ }^{\dagger}$, B. Stappers ${ }^{* *}$ and \\ G. Theureau* ${ }^{*}$ \\ ${ }^{*}$ Laboratoire de Physique et Chimie de l'Environnement (LPCE), CNRS-Orléans, France \\ ${ }^{\dagger}$ University of Manchester, Jodrell Bank Observatory, Cheshire, SK11 9DL, UK \\ ** Astronomical Institute 'Anton Pannekoek', University of Amsterdam, Kruislaan 403, 1098 SJ Amsterdam, The \\ Netherlands; Stichting ASTRON, Postbus 2, 7990 AA, Dwingeloo, The Netherlands \\ *GEPI, Paris Observatory, France
}

\begin{abstract}
PSR J1906+0746 is a highly relativistic binary pulsar, discovered with the P-ALFA survey in June 2005 [4]. Within a such system, it is expected to observe geodetic precession with a high precession rate. Possible first signs of pulse profile evolution were observed using archival data of the Parkes MultiBeam Survey and by Kasian et al. [3]. By combining the data from the Nançay and Jodrell Bank Radiotelescopes, we report on the clear detection of pulse profiles changes, a shift between both components of the pulse at a rate of $2.1^{\circ} \pm 0.1^{\circ}$ per year. However, only a marginal variation in the relative amplitude was found. We also noticed variations in the slope of the Polarisation Position Angle and in the circular polarisation in the main pulse area, using Nançay polarimetric data.
\end{abstract}

Keywords: pulsars; binary; geodetic precession; polarisation PACS: $97.60 . \mathrm{Gb}$

\section{INTRODUCTION}

The recent discovery of the binary pulsar PSR $\mathrm{J} 1906+0746(\mathrm{P}=144 \mathrm{~ms})$ in $3.98-\mathrm{hr}$ orbit immediately suggested that the geodetic precession could be observable in short timescale. This effect would cause a change in the longitude separation of the two main components, if we assume them to be the opposite side of a hollow cone, as the line of sight crosses a different part of the beam. Up to now, only 4 pulsars in binary systems exhibit changes in the mean pulse profile. A precession period of about 200 years following the standard geodetic precession formula is expected, depending on the nature of its companion. Furthermore, the no-detection of the secondary pulse in archival data from 1998 and an increase of the SNR for the main pulse between 1998 and 2005, suggested the first sign of change of beam orientation with respect to our line of sight [4]. A possible change in the profile was also noticed by Kasian et al. [3].

In the framework of the EPTA collaboration, we present the observations made with Nançay Radio Telescope (hereafter NRT) and the Lovell radiotelescope at the Jodrell Bank Observatory and quantify the profile changes.

\section{OBSERVATIONS}

The NRT is a meridian-type telescope, equivalent to a $94 \mathrm{~m}$ single dish, where maximum integration time is 1 hour per day. The observations were carried out from June 2005 , shortly after the discovery, to June 2007 , at a centre frequency of $1.4 \mathrm{Ghz}$. A bandwidth of $64 \mathrm{MHz}$ was coherently dedispersed by the Berkeley-OrleansNançay (BON) backend [1]. TEMPO was used to build a rotational model of the pulsar from the individuals 2 minutes TOAs. Higher SNR profiles can then be obtained for different integration times from minutes to weeks. A detailled timing analysis of the rotational behaviour of this pulsar will be presented in a forthcoming paper by Kasian et al.

Observations from the $76 \mathrm{~m}$ Lovell telescope were obtained at $1.4 \mathrm{Ghz}$ with a $64 \times 1 \mathrm{MHz}$ filterbank instrumentation. Lovell data are well complementary to Nançay ones, providing much longer integration times, from 30 minutes up to several hours, though with a sparser sampling.

\section{PULSE PROFILE CHANGES}

In a binary pulsar system, the angle between the spin axis and our line of sight will change if the pulsar spin axis is misaligned with respect to the orbital angular momentum [2]. Assuming a circular hollow-cone beam shape for 


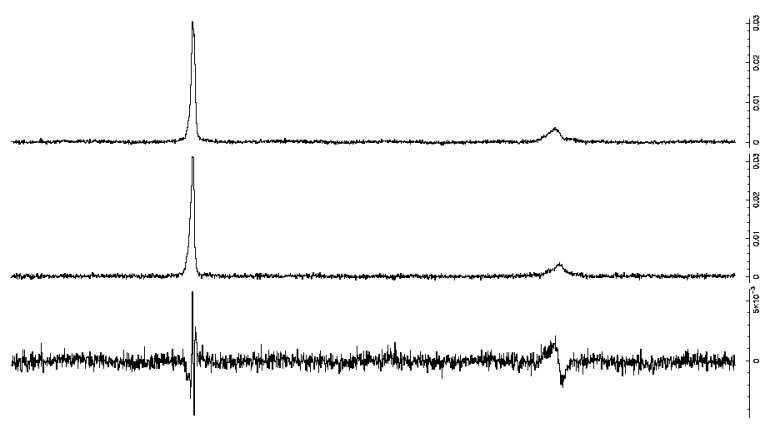

FIGURE 1. The top two profiles represent respectively the first and second year of the NRT observations where all subintegrations are realigned and folded using the rotational model. Substracting the second to the first, we build a difference profile shown at the bottom.

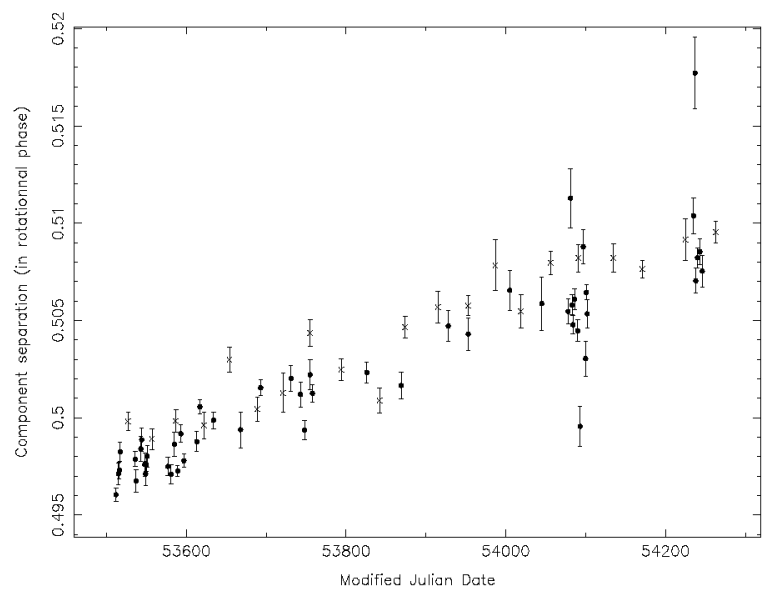

FIGURE 2. Component separation in terms of rotationnal phase agains MJD. The points indicate the NRT data, the crosses the JBO data. A drift of $2.1^{\circ} \pm 0.1^{\circ}$ per year was fitted to the data.

1906+0746, we expect to see a change in the components separation with time.

From 'equivalent SNR' profiles obtained on the first and the second year of observation, a 'difference' profile was built (see figure 1). A clear signature of a stretching, corresponding to a change of the component separation is seen.

We integrated observations from the NRT over one month, letting us with 21 monthly profiles. Including the Jodrell data, we measured the separation between the two components in terms of rotational phase. The result is shown in figure 2 . We fitted the points by a straight line and found a drift of $2.1^{\circ} \pm 0.1^{\circ}$ per year.

We also studied the amplitude ratio of the two components versus time (Figure 3 ). Because the Jodrell data are not dedispersed over $1 \mathrm{MHz}$, the narrow main pulse undergo a more important spreading than the broader second pulse, leading to a different amplitude ratio when

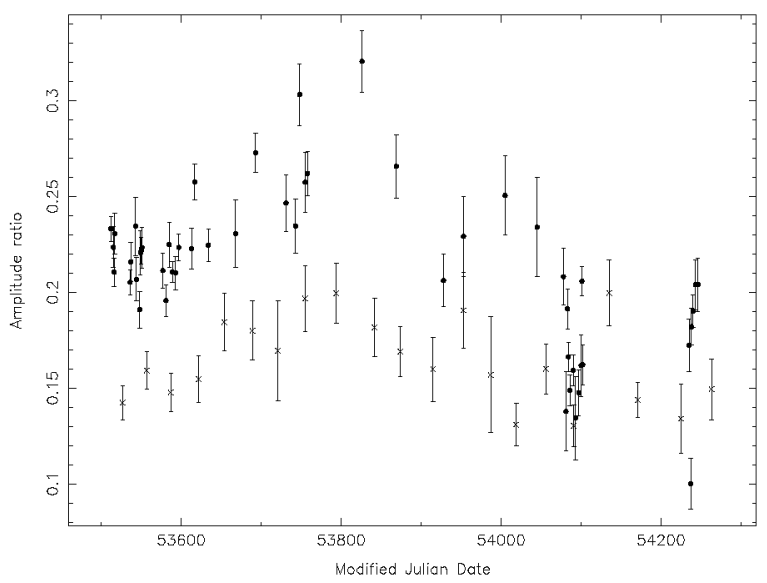

FIGURE 3. Amplitude ratio of the two components against MJD. The points indicate the NRT data, the cross the JBO data.

compared to coherent observations from the NRT. A marginal variation of the ratio can be seen in both datasets.

\section{POLARISATION CHANGES}

The classical model for pulsar polarimetry is the rotating vector model (RVM) by Radhakrishnan \& Cooke [5]. It predicts a characteristic S-shaped curve in the measured Position Angle (PA), when the emission beam sweeps past our line of sight. NRT data were integrated over 2 months to provide 11 high quality profiles. As seen in figure 4, small strucuture changes are observed in the upper part of the main component, as if a substructure was wandering around with a caroussel like behaviour.

In the bottom panels of figure 4 and figure 5, the slope of the PA is observed to slowly change. A tilt is even seen at the same time the profile seems to be depolarized. The circular polarisation, the dot-dash-dot line in figure 4 is also vanishing along the two years of observation.

\section{CONCLUSION}

A separation of the two components was observed at a rate of about $2.1^{\circ}$ per year using NRT and Jodrell data, as well as small structure changes for the main pulse. Nançay polarimetric data also indicate major polarisation changes. These changes detected in only two years of observation are probably due to geodetic precession, which manifest itself at short timescales as expected. A such system will hopefully permit to do a quick mapping of the radio beam in the near future. 


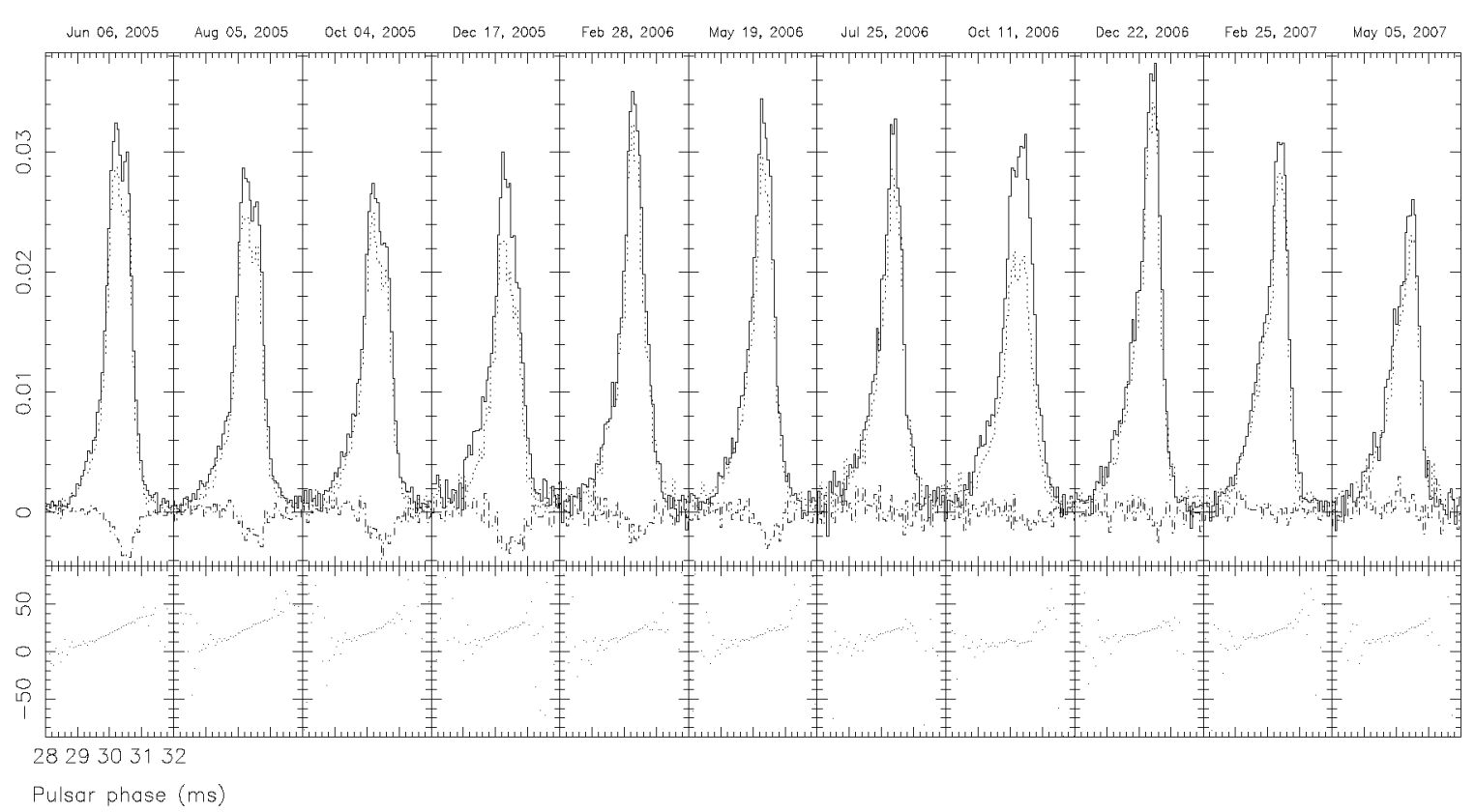

FIGURE 4. Nançay polarimetric data. Each inset represents a zoom (over $4 \mathrm{~ms}$ ) in the main pulse area. The full line represents the Total Intensity, the dotted line the linear polarisation and the dot-dash-dot line the circular polarisation. The polarisation position angle is shown in the lower panel.

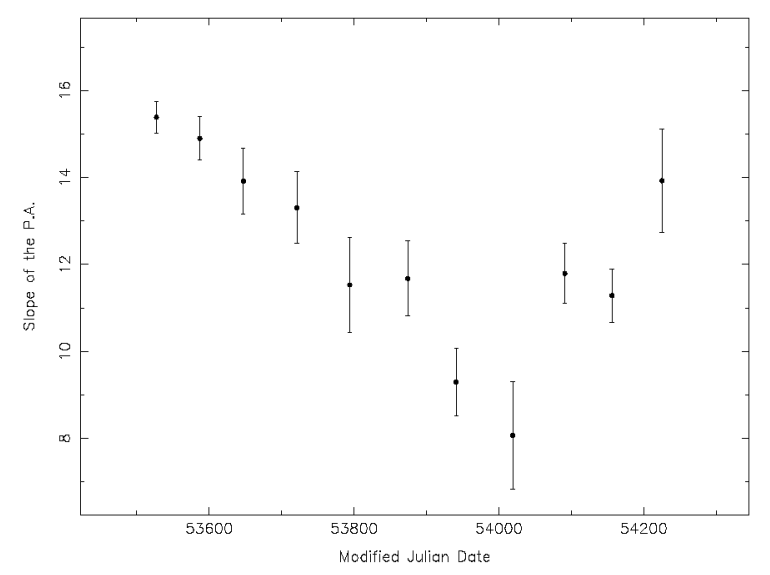

FIGURE 5. Slope of the position angle under the main pulse against MJD.

\section{REFERENCES}

1. I. Cognard and G. Theureau, On the Present and Future of Pulsar Astronomy, 26th meeting of the IAU, Joint Discussion 2, 16-17 August, 2006, Prague, Czech Republic, JD02, \#36, 2

2. T. Damour and R. Ruffini, 1974, C. R. Acad. Sc. Paris, Serie A, 279, 971

3. Kasian, L. E. \& Consortium, P. 2006, in American Astronomical Society Meeting Abstracts, 04.02

4. Lorimer et al. 2006, ApJ, 640, 428

5. Radhakrishnan, V. \& Cooke, D. J. 1969, Astrophys. Lett., 3,225 